\title{
Effects of irregularity and anisotropy on the propagation of shear waves
}

\author{
A. Chattopadhyay*, S. Gupta, V. K. Sharma and Pato Kumari \\ Department of Applied Mathematics, Indian School of Mines, Dhanbad- 826004, Jharkhand, INDIA \\ "Corresponding Author (e-mail: amares.c@gmail.com, A. Chattopadhyay)
}

\begin{abstract}
The propagation of shear waves differs between geo-media due to layer's structure and irregularity present in different layers. This paper studies the propagation of shear waves in a monoclinic layer with irregularity lying between two isotropic semiinfinite elastic medium. The displacement in the monoclinic layer is obtained by using perturbation technique. Then the dispersion relation is found in the assumed medium and is verified with the standard known results. Finally, effects of wave number and irregularity are studied numerically and the graphs are plotted for all cases. The dispersion curves for different size of irregularity are calculated and compared.
\end{abstract}

Keywords: Shear waves, intermediate monoclinic layer, irregular boundary, perturbation technique

\section{Introduction}

The study of earthquake is an important branch of seismology. It has revealed a great deal of information about how fracturing occurs in the earth and about strains and short-term deformation processes. The study of seismic waves allows us to make inferences about certain properties of the parts of the earth through which the waves have travelled as well as the source of waves.

Many problems in seismology can be solved by representing the Earth as a layered medium that is, formed by layers of certain thickness and mechanical properties. For certain problems we can use a flat approximation of parallel horizontal layers and they are reduced to two dimensions. Layer of constant properties may be considered as an approximation for media whose elastic coefficients vary in a continuous form with depth. Shear waves (SH waves) are the waves that is polarized so that its particle motion and direction of propagation are contained in a horizontal plane. Love waves, named after the British seismologist A. E. H. Love, who first predicted their existence in 1911, are characterized by horizontal motion normal to the direction of travel, with no vertical motion. In effect a Love wave is a polarized shear waves. Many authors have studied the propagation of Love waves, and different authors assumed different forms of irregularities at the interface. Chattopadhyay (1975) studied the effect of irregularities and non-homogeneities in the crustal layer on the propagation of Love waves. Bhattacharya (1962) considered the irregularity in the thickness of the transversely isotropic crustal layer. Mal (1962) derived the dispersion relation for Love waves due to abrupt thickening of the crustal layer. Ghosh (1961) discussed the propagation of Love waves across the oceanbed. Chattopadhyay and De (1983) studied the dispersion relation for Love waves in a non-dissipative liquid filled with porous solid underlain by an isotropic and homogeneous half-space. They derived the dispersion relation by applying the perturbation method and the phase velocity curve has been obtained for different irregularities by using the parameters of the porous medium, which were suggested by Biot (1961). Sinha (1967) studied the propagation of Love waves in a non-homogeneous stratum of finite depth sandwiched between two semi-infinite isotropic media. Sezawa (1935) discussed the propagation of Love waves generated from a buried source. Recently, Chattopadhyay et al. (2008) have studied SH waves propagation in a monoclinic layer over a semi-infinite elastic medium with irregularity. They have derived the dispersion relation for SH waves and shown the effect of irregularity in monoclinic medium.

The extension of earth is made up of solids, liquids and occluded gases. The solids are commonly called rocks and when minerals occur with definite geometrical outlines, they are called crystals. Crystals are solids bounded by natural plane surfaces or faces. A variety of crystal forms are possible and monoclinic form is one of them. The monoclinic system is the largest symmetry system with almost a third of all minerals belonging to one of its three classes. The motivation of the present problem is that the 
valuable materials, e.g., Lithium tantalate, Lithium niobate etc., which exhibits monoclinic symmetry are buried beneath the earth surface. These materials can be modeled as monoclinic materials. Xue (2000) studied the dielectric properties of Lithium tantalate and Lithium niobate. It is well known in the literature that the earth medium is not at all isotropic and homogeneous throughout, but it is anisotropic and inhomogeneous. Moreover, the discontinuities separating the different layers of the earth are not perfectly plane. Keeping these things in mind, we have considered the propagation of SH waves in a layered monoclinic medium lying between two elastic half-spaces. The irregularity has been taken in the monoclinic layer in the form of a rectangle. To solve the problem we have used the perturbation technique as indicated by Eringen and Samuels (1959). It is shown that the phase velocity of Love waves depends not only on the wave number and depth of the irregularity but layer structure also.

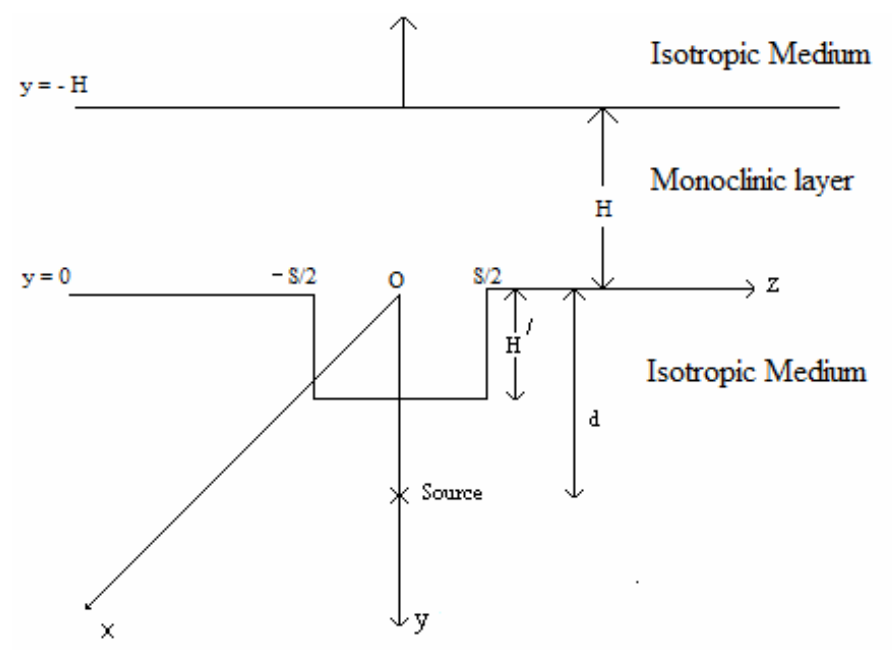

Figure 1: Geometry of the problem

\section{Formulation of the problem}

We choose the $y$-axis vertically downwards and $z$-axis along the interface between the lower semi-infinite medium and the monoclinic layer (Figure 1). We assume the irregularity in the form of a rectangle with length s and depth $\mathrm{H}^{\prime}$. The origin is placed at the middle point of the interface irregularity. $H$ is the thickness of the layer. Source of the disturbance is placed on positive $y$ axis at a distance $d\left(>H^{\prime}\right)$ from the origin.

The interface between the layer and lower half-space is given by

$y=\varepsilon h(z)$

where $h(z)=\left\{\begin{array}{cc}0 & \text { for } z \leq-\frac{s}{2}, z \geq \frac{s}{2} \\ f(z) & \text { for }-\frac{s}{2} \leq z \leq \frac{s}{2}\end{array}\right.$,

$\varepsilon=\frac{H^{\prime}}{S}<<1$. The function $f(z)$ describes the shape of the irregularity. For present paper we have taken the rectangular irregularity with $f(z)=s$, but the results obtained can be utilized for other shape of irregularity as well. Let us take $\mu_{r}, \rho_{r}, u_{r}$ $(r=1,2,3)$ as the rigidities, densities and displacements components of the upper medium, monoclinic layer and lower media respectively.

\section{Equations of motion and boundary conditions}

For waves propagating in the z-direction and causing displacement in the x-direction only, we assume that $u=u(y, z, t), v=0, w=0$.

The equation of motion in the intermediate monoclinic layer is 
$\frac{\partial^{2} u_{2}}{\partial y^{2}}+2 \frac{C_{56}}{C_{66}} \frac{\partial^{2} u_{2}}{\partial y \partial z}+\frac{C_{55}}{C_{66}} \frac{\partial^{2} u_{2}}{\partial z^{2}}=\frac{1}{\beta_{2}^{2}} \frac{\partial^{2} u_{2}}{\partial t^{2}}$

where $C_{55}, C_{56}, C_{66}$ are elastic coefficients for monoclinic crystalline medium under the given geometry.

Similarly the equations of motion for upper and lower semi-infinite mediums are

$\frac{\partial^{2} u_{1}}{\partial y^{2}}+\frac{\partial^{2} u_{1}}{\partial z^{2}}=\frac{1}{\beta_{1}^{2}} \frac{\partial^{2} u_{1}}{\partial t^{2}}$

$\frac{\partial^{2} u_{3}}{\partial y^{2}}+\frac{\partial^{2} u_{3}}{\partial z^{2}}=\frac{1}{\beta_{3}^{2}} \frac{\partial^{2} u_{3}}{\partial t^{2}}$

where $\beta_{1}=\sqrt{\frac{\mu_{1}}{\rho_{1}}}, \beta_{2}=\sqrt{\frac{C_{66}}{\rho_{2}}}, \beta_{3}=\sqrt{\frac{\mu_{3}}{\rho_{3}}}$.

The boundary conditions are as follows:

i) $u_{1}=u_{2}$ at $y=-H$,

$u_{2}=u_{3}$ at $y=\varepsilon h(z)$

ii) $\mu_{1} \frac{\partial u_{1}}{\partial y}=C_{56} \frac{\partial u_{2}}{\partial z}+C_{66} \frac{\partial u_{2}}{\partial y}$ at $y=-H$,

$$
\frac{\partial u_{2}}{\partial y}\left[C_{66}-C_{56} \varepsilon h^{\prime}\right]+\left[C_{56}-C_{55} \varepsilon h^{\prime}\right] \frac{\partial u_{2}}{\partial z}=\mu_{3}\left[\frac{\partial u_{3}}{\partial y}-\varepsilon h^{\prime} \frac{\partial u_{3}}{\partial z}\right] \text { at } y=\varepsilon h(z),
$$

where $h^{\prime}=\frac{d h(z)}{d z}$.

\section{Solution of the problem}

Let us consider the time-dependent displacements $u_{j}(y, z, t)=U_{j}(y, z) e^{i \omega t}(j=1,2,3 ; i=\sqrt{-1})$, where $\omega$ is the angular frequency, then the Eqs. (2) and (3) become

$\frac{\partial^{2} U_{1}}{\partial y^{2}}+\frac{\partial^{2} U_{1}}{\partial z^{2}}+\frac{\omega^{2}}{\beta_{1}^{2}} U_{1}=0$

$\frac{\partial^{2} U_{2}}{\partial y^{2}}+2 \frac{C_{56}}{C_{66}} \frac{\partial^{2} U_{2}}{\partial y \partial z}+\frac{C_{55}}{C_{66}} \frac{\partial^{2} U_{2}}{\partial z^{2}}+\frac{\omega^{2}}{\beta_{2}^{2}} U_{2}=0$

$\frac{\partial^{2} U_{3}}{\partial y^{2}}+\frac{\partial^{2} U_{3}}{\partial z^{2}}+\frac{\omega^{2}}{\beta_{3}^{2}} U_{3}=0$.

Taking the Fourier transform $\bar{U}_{r}(y, \eta)$ of $U_{r}(y, z)$ (vide Appendix-A [Eq. (A1]) of Eqs. (5), (6), and (7), we obtain the reduced system of equations as

$\frac{d^{2} \bar{U}_{1}}{d y^{2}}-p_{1}^{2} \bar{U}_{1}=0$

$\frac{d^{2} \bar{U}_{2}}{d y^{2}}+a \frac{d \bar{U}_{2}}{d y}+p^{2} \bar{U}_{2}=0$

$\frac{d^{2} \bar{U}_{3}}{d y^{2}}-p_{3}^{2} \bar{U}_{3}=0$,

where $p_{1}, p, p_{3}$, and $a$ are defined in the Appendix-A, [Eq. (A2)].

Appropriate solution of Eqs. (8), (9), and (10) are

$\bar{U}_{1}(y, \eta)=D e^{p_{1} y}, \quad y \leq-H$ 
$\begin{array}{lrl}\bar{U}_{2}(y, \eta) & =e^{-\frac{a}{2} y}\left(A \cos p_{2} y+B \sin p_{2} y\right), & -H \leq y \leq \varepsilon h(z) \\ \bar{U}_{3}(y, \eta)=C e^{-p_{3} y} & y \geq \varepsilon h(z)\end{array}$

where $p_{2}$ is defined in the Appendix-A [Eq. (A3)].

The displacements in the three media are

$U_{1}(y, z)=\frac{1}{2 \pi} \int_{-\infty}^{\infty} D e^{p_{1} y} e^{-i \eta z} d \eta$,

$U_{2}(y, z)=\frac{1}{2 \pi} \int_{-\infty}^{\infty} e^{-\frac{a}{2} y}\left(A \cos p_{2} y+B \sin p_{2} y\right) e^{-i \eta z} d \eta$,

and $U_{3}(y, z)=\frac{1}{2 \pi} \int_{-\infty}^{\infty}\left(C e^{-p_{3} y}+\frac{2}{p_{3}} e^{p_{3} y} e^{-p_{3} d}\right) e^{-i \eta z} d \eta$

where the second term in the integrand of $U_{3}$ is introduced due to the source in the lower medium (Sezawa, 1935). We use the perturbation method given by Eringen and Samuels (1959), to set the following approximations due to small value of $\varepsilon$

$$
A \cong A_{0}+A_{1} \varepsilon, B \cong B_{0}+B_{1} \varepsilon, C \cong C_{0}+C_{1} \varepsilon, D \cong D_{0}+D_{1} \varepsilon, e^{ \pm v \varepsilon h} \cong 1 \pm v \varepsilon h
$$

where $v=a / 2$. Since the boundary is not uniform the terms $\mathrm{A}, \mathrm{B}, \mathrm{C}$, and $\mathrm{D}$ in Eq. (17) are also functions of $\varepsilon$. Expanding these terms in ascending powers of $\varepsilon$ and keeping in view that $\varepsilon$ is small and so retaining the terms up to the first order of $\varepsilon$, A, B, C, and $\mathrm{D}$ can be approximated as in Eq. (17). In physical situations, when the depth $\mathrm{H}^{\prime}$ of the irregular boundary is too small with respect to the length of the boundary s, the above assumptions are justified.

Defining the Fourier transform $\bar{h}(\lambda)$ of $h(z)$ as

$\bar{h}(\lambda)=\int_{-\infty}^{\infty} h(z) e^{i \lambda z} d z$

and so, $h(z)=\frac{1}{2 \pi} \int_{-\infty}^{\infty} \bar{h}(\lambda) e^{-i \lambda z} d \lambda$.

Therefore $h^{\prime}(z)=-\frac{i}{2 \pi} \int_{-\infty}^{\infty} \lambda \bar{h}(\lambda) e^{-i \lambda z} d \lambda$.

Using boundary conditions (4a) to (4d) along with Eqs. (14) to (17), we obtain the following eight equations (after equating the coefficient of $\varepsilon$ and the absolute term):

$$
\begin{aligned}
& -e^{\frac{1}{2} a H} \cos \left(p_{2} H\right) A_{0}+e^{\frac{1}{2} a H} \sin \left(p_{2} H\right) B_{0}+e^{-p_{1} H} D_{0}=0, \\
& -e^{\frac{1}{2} a H} \cos \left(p_{2} H\right) A_{1}+e^{\frac{1}{2} a H} \sin \left(p_{2} H\right) B_{1}+e^{-p_{1} H} D_{1}=0, \\
& \left\{i k C_{56} \cos p_{2} H+\frac{a}{2} C_{66} \cos p_{2} H-p_{2} C_{66} \sin p_{2} H\right\} A_{0}- \\
& -\left\{i k C_{56} \sin p_{2} H+C_{66}\left(\frac{a}{2} \sin p_{2} H+p_{2} \cos p_{2} H\right)\right\} B_{0}+D_{0} u_{1} p_{1} e^{-p_{1} H} e^{-\frac{a}{2} H}=0, \\
& \left\{i k C_{56} \cos p_{2} H+\frac{a}{2} C_{66} \cos p_{2} H-p_{2} C_{66} \sin p_{2} H\right\} A_{1}- \\
& -\left\{i k C_{56} \sin p_{2} H+C_{66}\left(\frac{a}{2} \sin p_{2} H+p_{2} \cos p_{2} H\right)\right\} B_{1}+D_{1} u_{1} p_{1} e^{-p_{1} H} e^{-\frac{a}{2} H}=0, \\
& A_{0}-C_{0}-\frac{2}{p_{3}} e^{-p_{3} d}=0,
\end{aligned}
$$




$$
\begin{aligned}
& A_{1}-C_{1}=R_{1}(k), \\
& \left(C_{66} \frac{a}{2}+i k C_{56}\right) A_{0}-p_{2} C_{66} B_{0}-\mu_{3} p_{3} C_{0}=-2 \mu_{3} e^{-p_{3} d}, \\
& \left(C_{66} \frac{a}{2}+i k C_{56}\right) A_{1}-p_{2} C_{66} B_{1}-\mu_{3} p_{3} C_{1}=R_{2}(k)
\end{aligned}
$$

where $R_{1}(k)$ and $R_{2}(k)$ are given in the Appendix-A [Eqs. (A4) and (A5)].

Solving the above eight equations, we obtain the values of $A_{0}, B_{0}, C_{0}, D_{0}, A_{1}, B_{1}, C_{1}, D_{1}$ which are given in the Appendix-A [Eq. (A6)].

The displacement in the monoclinic layer will be

$$
\begin{aligned}
& U_{2}=\frac{1}{2 \pi} \int_{-\infty}^{\infty} \frac{-2}{E(k) \cos \left(p_{2} H\right)}\left[4 \mu_{3}+\varepsilon\left(\mu_{3} p_{3} R_{1}-R_{2}\right) e^{p_{3} d}\right] \times \\
& \times\left\{\left(2 \cos \left(p_{2} y\right) \sin \left(p_{2} H\right) \mu_{1} p_{1}+2 i \cos \left(p_{2} y\right) C_{56} k \sin \left(p_{2} H\right)+\cos \left(p_{2} y\right) C_{66} a \sin \left(p_{2} H\right)+\right.\right. \\
& \quad 2 \cos \left(p_{2} y\right) \cos \left(p_{2} H\right) C_{66} p_{2}+2 \sin \left(p_{2} y\right) \cos \left(p_{2} H\right) \mu_{1} p_{1}+2 i \sin \left(p_{2} y\right) C_{56} k \cos \left(p_{2} H\right)+ \\
&\left.\left.\sin \left(p_{2} y\right) C_{66} a \cos \left(p_{2} \mathrm{H}\right)-2 \sin \left(p_{2} y\right) C_{66} \sin \left(p_{2} \mathrm{H}\right) \mathrm{p}_{2}\right) \mathrm{e}^{-\left(\mathrm{p}_{3} \mathrm{~d}+\frac{1}{2} \mathrm{ay}\right)}\right\} e^{-i k z} d k .
\end{aligned}
$$

Now from Eqs. (1) and (18), we have

$$
\bar{h}(\lambda)=\frac{2 s}{\lambda} \sin \frac{\lambda s}{2} \text {. }
$$

Using Eqs. (A4) and (A5) defined in Appendix-A, we get

$\mu_{3} p_{3} R_{1}-R_{2}=\frac{s}{\pi} \int_{-\infty}^{\infty}[\varphi(k-\lambda)+\varphi(k+\lambda)] \frac{1}{\lambda} \sin \frac{\lambda s}{2} d \lambda$

where $\varphi(k-\lambda)$ is given in Appendix-A [Eq. (A7)]. Using asymptotic formula of Willis (1948), Tranter (1966) and neglecting the terms containing $2 / \mathrm{s}$ and higher powers of $2 / \mathrm{s}$ for large $\mathrm{s}$, we have

$$
\int_{-\infty}^{\infty}[\varphi(k-\lambda)+\varphi(k+\lambda)] \frac{1}{\lambda} \sin \frac{\lambda s}{2} d \lambda=\frac{\pi}{2} 2 \varphi(k)=\pi \varphi(k) .
$$

Using Eqs. (29) and (30), we obtain

$$
\mu_{3} p_{3} R_{1}-R_{2}=s \varphi(k)=\frac{H^{\prime}}{\varepsilon} \varphi(k) \text {. }
$$

Therefore the displacement in the monoclinic layer is

$$
\begin{aligned}
& U_{2}=-\frac{1}{2 \pi} \int_{-\infty}^{\infty} \frac{8 \mu_{3}}{E(k) \cos p_{2} H\left[1-H^{\prime} \psi(k) e^{p_{3} d}\right]} \times \\
&\left\{\left(2 \cos \left(p_{2} y\right) \sin \left(p_{2} H\right) \mu_{1} p_{1}+2 i \cos \left(p_{2} y\right) C_{56} k \sin \left(p_{2} H\right)+\cos \left(p_{2} y\right) C_{66} a \sin \left(p_{2} H\right)+\right.\right. \\
& 2 \cos \left(p_{2} y\right) \cos \left(p_{2} H\right) C_{66} p_{2}+2 \sin \left(p_{2} y\right) \cos \left(p_{2} H\right) \mu_{1} p_{1}+2 i \sin \left(p_{2} y\right) C_{56} k \cos \left(p_{2} H\right)+ \\
&\left.\left.\sin \left(p_{2} y\right) C_{66} a \cos \left(p_{2} H\right)-2 \sin \left(p_{2} y\right) C_{66} \sin \left(p_{2} H\right) p_{2}\right) e^{-\left(2 p_{3} d+a y\right) / 2}\right\} e^{-i k z} d k .
\end{aligned}
$$

where $\psi(k)=\frac{\phi(k)}{4 \mu_{3}}$

The value of this integral will depend entirely on the contribution of the poles of the integrand. The poles are located at the roots of equation

$$
E(k) \cos p_{2} H\left[1-H^{\prime} \psi(k) e^{p_{3} d}\right]=0 .
$$

This equation was examined in the study of shear waves (cf. Achenbach, 1976, p - 293). 


\section{Dispersion relation}

If $\mathrm{c}$ is the common wave velocity of the wave propagating along the surface, then writing $\omega=c k$ and replacing $p_{1}=k P_{1}, \quad p_{2}=k P_{2}, a=-2 i k C_{56} / C_{66}$ and $p_{3}=k P_{3}$ in Eq. (32)

(where $\omega$ is the angular frequency and $P_{1}, P_{2}, P_{3}$ are defined in Appendix-B [Eq. (B1)].), and solving Eq. (32) we get

$\tan \left\{\sqrt{\frac{c^{2}}{\beta_{2}^{2}}-\frac{C_{55}}{C_{66}}+\frac{C_{56}^{2}}{C_{66}^{2}}} \frac{k H}{2}\right\}=\frac{\left(\xi_{7}+i \xi_{8}\right)+\sqrt{\xi_{9}+i \xi_{10}}}{\xi_{11}+i \xi_{12}}$

where symbols on right hand side are defined in Appendix-B [Eq. (B2)].

Equating both the real and imaginary part we get

$$
\begin{aligned}
& \tan \left\{\sqrt{\frac{c^{2}}{\beta_{2}^{2}}-\frac{C_{55}}{C_{66}}+\frac{C_{56}^{2}}{C_{66}^{2}}} \frac{k H}{2}\right\}=\frac{\left(\xi_{7}+\xi_{13}\right) \xi_{11}+\left(\xi_{8}+\xi_{14}\right) \xi_{12}}{\xi_{11}{ }^{2}+\xi_{12}{ }^{2}} \\
& \text { and } \frac{\left(\xi_{8}+\xi_{14}\right) \xi_{11}-\left(\xi_{7}+\xi_{13}\right) \xi_{12}}{\xi_{11}{ }^{2}+\xi_{12}{ }^{2}}=0
\end{aligned}
$$

where $\xi_{11}$ and $\xi_{12}$ are given in Appendix-B [Eq. (B2)].

We consider the real part i.e. Eq. (33) which gives the dispersion relation of Love wave in a monoclinic layer with rectangular irregularity between two isotropic half spaces

If we put $C_{55}=C_{66}=\mu_{2}, C_{56}=0$, in Eq. (33) and equating the real part, we have

$\tan \left\{\sqrt{\frac{c^{2}}{\beta_{2}^{2}}-1} \frac{k H}{2}\right\}=\frac{S_{1}^{\prime}}{T_{1}^{\prime}} \quad$ (cf. Chattopadhyay (1975))

where $S_{1}^{\prime}$ and $T_{1}^{\prime}$ are given in Appendix-B [Eq. (B3)].

Eq. (34) gives the dispersion relation of Love wave in an isotropic layer with rectangular irregularity between two isotropic half spaces

If we further take $\mathrm{H}^{\prime}=0$ (i.e. no irregularity), then Eq. (34) becomes

$\tan \left\{\sqrt{\frac{c^{2}}{\beta_{2}^{2}}-1} k H\right\}=\frac{\mu_{2} \sqrt{\frac{c^{2}}{\beta_{2}^{2}}-1}\left\{\mu_{1} \sqrt{1-\frac{c^{2}}{\beta_{1}^{2}}}+\mu_{3} \sqrt{1-\frac{c^{2}}{\beta_{3}^{2}}}\right\}}{\mu_{2}^{2}\left\{\frac{c^{2}}{\beta_{2}^{2}}-1\right\}-\mu_{1} \mu_{3} \sqrt{1-\frac{c^{2}}{\beta_{1}^{2}}} \sqrt{1-\frac{c^{2}}{\beta_{3}^{2}}}}$.

Equation (35) is the standard dispersion relation of SH waves in three isotropic media. The roots of this dispersion relation are real if either $\beta_{2}<c<\beta_{1} \leq \beta_{3}$ or $\beta_{2}<c<\beta_{3} \leq \beta_{1}$, which is also the necessary condition for Love type waves to exist.

\section{Numerical calculations and discussions}

From Eq. (31) we find the displacement in the intermediate monoclinic layer. Both the Eqs. (33) and (34) give the resulting dispersion relation for three-layer problem under two different conditions. For graphical representation of phase velocity in a monoclinic layer between two isotropic media, we take the following data:

(i) The density and rigidity for upper isotropic homogeneous medium are (Gubbins, 1990)

$$
\rho_{1}=3293 \mathrm{Kg} / \mathrm{m}^{3}, \mu_{1}=7.45 \times 10^{10} \mathrm{~N} / \mathrm{m}^{2}
$$

(ii) The material constants for Lithium tantalate which exhibit monoclinic symmetry are (Tiersten, 1969)

$$
C_{55}=0.94 \times 10^{11} \mathrm{~N} / \mathrm{m}^{2}, C_{56}=-0.11 \times 10^{11} \mathrm{~N} / \mathrm{m}^{2}, C_{66}=0.93 \times 10^{11} \mathrm{~N} / \mathrm{m}^{2}, \rho_{2}=7450 \mathrm{~kg} / \mathrm{m}^{3} \text {. }
$$

(iii) The density and rigidity for lower isotropic homogeneous medium are (Gubbins, 1990)

$$
\rho_{3}=3535 \mathrm{~kg} / \mathrm{m}^{3}, \mu_{3}=7.84 \times 10^{10} \mathrm{~N} / \mathrm{m}^{2} \text {. }
$$


We have shown in Figure 2, the variation in dimensionless phase velocity $c / \beta_{2}$ against dimensionless wave number $k H$ for a layered monoclinic medium lying between two isotropic half-space for different values of $H^{\prime} / H$ (i.e. ratio of irregularity depth to layer width).

For graphical representation of phase velocity in a layered isotropic medium lying between two isotropic half-space, we have considered the following data:

(i) Upper isotropic homogeneous medium (Gubbins, 1990)

$$
\rho_{1}=3293 \mathrm{~kg} / \mathrm{m}^{3}, \quad \mu_{1}=7.45 \times 10^{10} \mathrm{~N} / \mathrm{m}^{2} \text {. }
$$

(ii) Intermediate isotropic layer (Gubbins, 1990)

$$
\rho_{2}=3364 \mathrm{~kg} / \mathrm{m}^{3}, \quad \mu_{2}=6.34 \times 10^{10} \mathrm{~N} / \mathrm{m}^{2} .
$$

(iii) Lower isotropic homogeneous medium (Gubbins, 1990)

$$
\rho_{3}=3535 \mathrm{~kg} / \mathrm{m}^{3}, \quad \mu_{3}=7.84 \times 10^{10} \mathrm{~N} / \mathrm{m}^{2} .
$$

Figure 3 shows the variation in dimensionless phase velocity $c / \beta_{2}$ against dimensionless wave number $k H$.

It is clear from both Figures (2) and (3) that phase velocity decreases with increase in wave number. Also increase in ratio $H^{\prime} / H$ results in lower phase velocity corresponding to a fixed value of wave number. It is interesting to note that $\left(c / \beta_{2}\right)_{\frac{H^{\prime}}{H}=0} \geq\left(c / \beta_{2}\right)_{\frac{H^{\prime}}{H} \neq 0}$ in both the cases. It is also found that the impact of $H^{\prime} / H$ on phase velocity becomes negligible for higher values of $H^{\prime} / H$ in monoclinic layer, but for isotropic layer this impact is more visible with higher value of $H^{\prime} / H$.

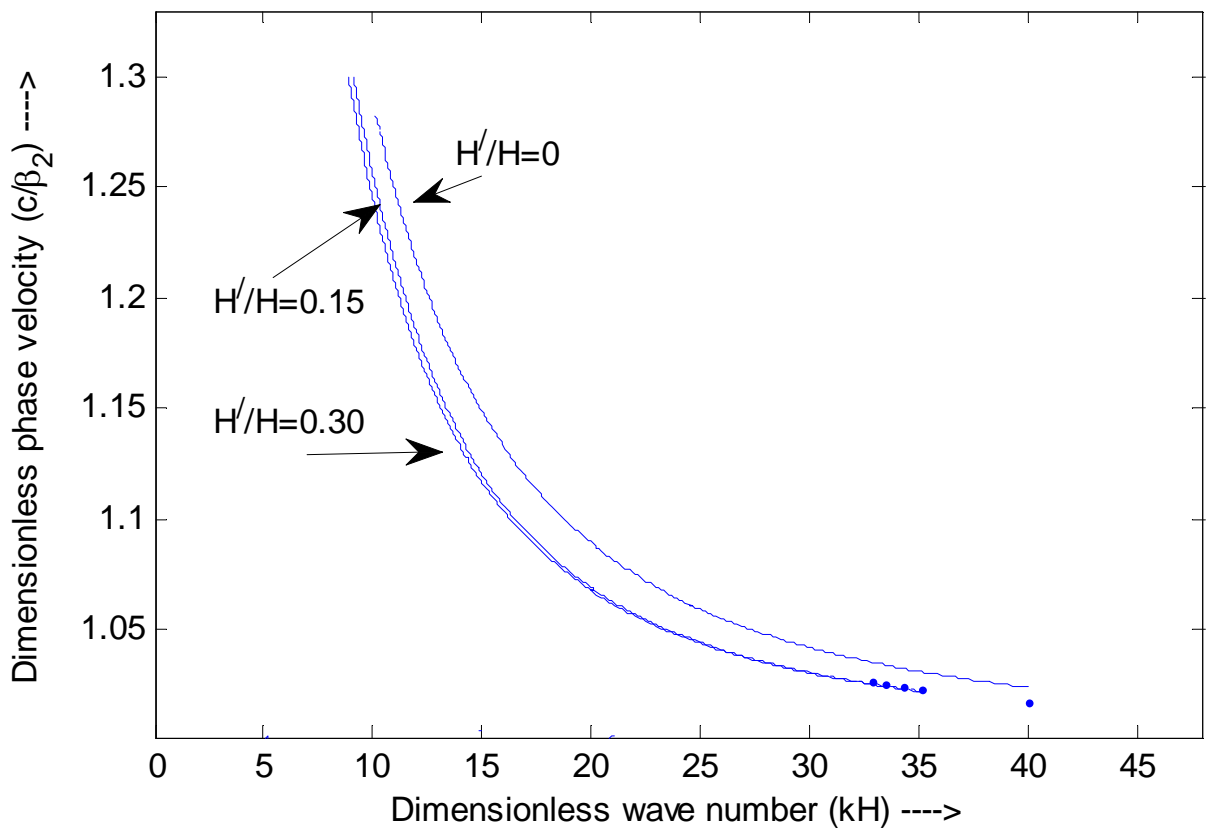

Figure 2: Variation of $c / \beta_{2}$ against $k H$ in a monoclinic layer lying between two isotropic semi-infinite media for different value of $H^{\prime} / H(=0,0.15,0.30)$. 


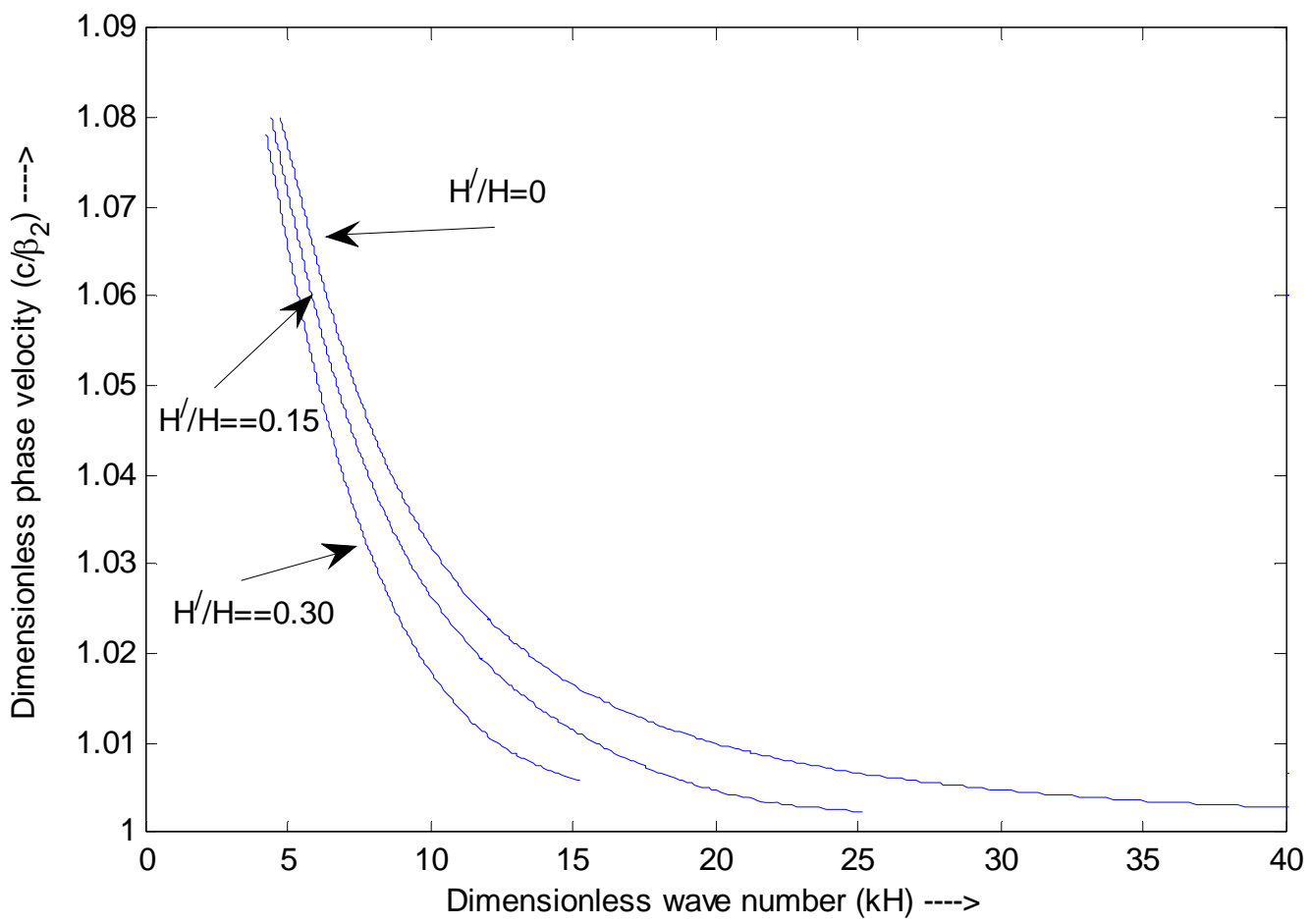

Figure 3: Variation of $c / \beta_{2}$ against $k H$ in an isotropic layer lying between two isotropic semi-infinite media for different value of $H^{\prime} / H(=0,0.15,0.30)$.

\section{Conclusions}

Propagation of shear waves in a monoclinic layer with irregular boundary sandwiched between two semi-infinite isotropic half spaces has been studied. The Eringen's perturbation method is applied to find the displacement field in the layer. The result obtained is used to get the dispersion relation in an irregular monoclinic layer. The dispersion relation for the isotropic layer with and without irregularity, between semi-infinite isotropic half spaces has been derived as a special case of the present problem. The effect of dimensionless wave number on dispersion curve is found numerically and shown graphically for both the monoclinic and for isotropic layer. Variation of phase velocity for different ratio of irregularity depth to layer width is studied and shown graphically. From above discussion we conclude that:

1. In general the phase velocity of Shear waves in a monoclinic or isotropic layer with irregularity, between semi-infinite isotropic half spaces decreases with increase in wave number.

2. Impact of ratio of irregularity depth to layer width is different for monoclinic and isotropic layer.

3. Phase velocity is a function of wave number as well as layer width and depth of irregularity.

4. Increase in depth of the irregularity decrease the magnitude of phase velocity.

Thus it can be concluded that the phase velocity in a layer with irregularity between two half spaces is affected by not only the shape of irregularity but also by wave number, the ratio of the depth of the irregularity to layer width and layer structure.

\section{Acknowledgement}

Authors are grateful to the reviewer for suggesting enormous improvements in the paper. The authors also convey their sincere thanks to Indian School of Mines, Dhanbad and DST, New Delhi for providing financial support through Project No. SR/S4/MS: 436/07, Project title: "Wave propagation in anisotropic media".

\section{$\underline{\text { Appendix A }}$}

The Fourier transform $\bar{U}_{r}(y, \eta)=\int_{-\infty}^{\infty} U_{r}(y, z) e^{i \eta z} d z$. 


$$
\begin{aligned}
& \text { and so } U_{r}(y, z)=\frac{1}{2 \pi} \int_{-\infty}^{\infty} \bar{U}_{r}(y, \eta) e^{-i \eta z} d \eta \\
& \begin{array}{c}
p_{1}^{2}=\eta^{2}-\frac{\omega^{2}}{\beta_{1}^{2}}, a=-2 i \eta \frac{C_{56}}{C_{66}}, p^{2}=\frac{\omega^{2}}{\beta_{2}^{2}}-\frac{C_{55}}{C_{66}} \eta^{2}, p_{3}^{2}=\eta^{2}-\frac{\omega^{2}}{\beta_{3}^{2}} . \\
p_{2}=\sqrt{p^{2}-\frac{a^{2}}{4}}=\left(\frac{\omega^{2}}{\beta_{2}^{2}}-\frac{C_{55}}{C_{66}} \eta^{2}+\eta^{2} \frac{C_{56}^{2}}{C_{66}^{2}}\right)^{1 / 2} \\
R_{1}(k)=\frac{1}{2 \pi} \int_{-\infty}^{\infty}\left[\frac{a}{2} A_{0}-B_{0} p_{2}-C_{0} p_{3}+2 e^{-p_{3} d}\right]^{\eta=k-\lambda} \bar{h}(\lambda) d \lambda, \\
R_{2}(k)=\frac{1}{2 \pi} \int_{-\infty}^{\infty}\left[\left\{C_{56} B_{0} p_{2}+\frac{2 i}{p_{3}} \mu_{3} k e^{-p_{3} d}+\mu_{3} k C_{0} i-\frac{1}{2} C_{56} A_{0} a-i k C_{55} A_{0}\right\} i \lambda+\right. \\
\left\{-A_{0} p_{2}^{2} C_{66}-\mu_{3} C_{0} p_{3}^{2}-2 \mu_{3} p_{3} e^{-p_{3} d}-i k C_{56} B_{0} p_{2}+\frac{i k}{2} C_{56} A_{0} a+\right. \\
\left.\left.\left.\quad+\frac{1}{4} C_{66} a^{2} A_{0}\right)-C_{66} a B_{0} p_{2}\right\}\right]^{\eta=k-\lambda} \bar{h}(\lambda) d \lambda .
\end{array}
\end{aligned}
$$

$$
\begin{aligned}
& A_{0}=-\frac{8 \mu_{3}\left(2 \mu_{1} p_{1} \tan \left(p_{2} H\right)+2 i C_{56} k \tan \left(p_{2} H\right)+C_{66} a \tan \left(p_{2} H\right)+2 C_{66} p_{2}\right)}{e^{p_{3} d} E(k)}, \\
& B_{0}=-\frac{8 \mu_{3}\left(2 \mu_{1} p_{1}+2 i C_{56} k+C_{66} a-2 C_{66} \tan \left(p_{2} H\right) p_{2}\right)}{e^{p_{3} d} E(k)}, \\
& C_{0}=\frac{\left(-2 \xi_{1} p_{1}+\xi_{2}+\xi_{3}\right)}{p_{3} e^{p_{3} d} E(k)}, D_{0}=\frac{-16 C_{66} \mu_{3} p_{2} e^{\left(p_{1} H-p_{3} d+\frac{1}{2} a H\right)}}{\cos \left(p_{2} H\right) E(k)}, \\
& \xi_{1}=4 \mu_{1} \tan \left(\mathrm{p}_{2} \mathrm{H}\right) \mu_{3} \mathrm{p}_{3}+2 \mu_{1} \tan \left(\mathrm{p}_{2} \mathrm{H}\right) C_{66} \mathrm{a}+4 \mathrm{i} \mu_{1} \tan \left(\mathrm{p}_{2} \mathrm{H}\right) \mathrm{k} C_{56}-4 \mu_{1} C_{66} \mathrm{p}_{2} \text {, } \\
& \xi_{2}=-2 C_{66}^{2} \mathrm{a}^{2} \tan \left(\mathrm{p}_{2} \mathrm{H}\right)-8 \mathrm{i} C_{56} \mathrm{ktan}\left(\mathrm{p}_{2} \mathrm{H}\right) \mu_{3} \mathrm{p}_{3}-4 C_{66} \operatorname{atan}\left(\mathrm{p}_{2} \mathrm{H}\right) \mu_{3} \mathrm{p}_{3}-8 C_{66} \mathrm{p}_{2} \mu_{3} \mathrm{p}_{3}, \\
& \xi_{3}=8 C_{56}^{2} \mathrm{k}^{2} \tan ^{2}\left(\mathrm{p}_{2} \mathrm{H}\right)-8 \mathrm{i} C_{56} \mathrm{ktan}\left(\mathrm{p}_{2} \mathrm{H}\right) C_{66} \mathrm{a}-8 C_{66}^{2} \tan \left(\mathrm{p}_{2} \mathrm{H}\right) \mathrm{p}_{2}^{2} \text {, } \\
& A_{1}=\frac{-2\left(2 \sin \left(p_{2} H\right) \mu_{1} \mathrm{p}_{1}+2 \mathrm{ik} C_{56} \sin \left(p_{2} H\right)+C_{66} \operatorname{asin}\left(p_{2} H\right)+2 C_{66} \cos \left(p_{2} H\right) p_{2}\right)\left(\mu_{3} \mathrm{p}_{3} \mathrm{R}_{1}-\mathrm{R}_{2}\right)}{\cos \left(p_{2} H\right) E(k)}, \\
& B_{1}=\frac{-2\left(2 \cos \left(p_{2} H\right) \mu_{1} \mathrm{p}_{1}+2 \mathrm{ik} C_{56} \cos \left(p_{2} H\right)+C_{66} \mathrm{acos}\left(p_{2} H\right)-2 C_{66} \sin \left(p_{2} H\right) p_{2}\right)\left(\mu_{3} \mathrm{p}_{3} \mathrm{R}_{1}-\mathrm{R}_{2}\right)}{\cos \left(p_{2} H\right) E(k)}, \\
& C_{1}=\frac{-\left(\xi_{4}+\xi_{5}+\xi_{6}\right)}{\cos \left(p_{2} H\right) E(k)}, D_{1}=\frac{-4 C_{66} \mathrm{p}_{2}\left(\mu_{3} \mathrm{p}_{3} \mathrm{R}_{1}-\mathrm{R}_{2}\right) \mathrm{e}^{\mathrm{H}\left(\mathrm{a}+2 \mathrm{p}_{1}\right) / 2}}{E(k)}, \\
& \xi_{4}=-4 \sin \left(p_{2} H\right) \mu_{1} \mathrm{p}_{1} \mathrm{R}_{2}+4 \mathrm{i} \mu_{1} \mathrm{p}_{1} \mathrm{R}_{1} \sin \left(p_{2} H\right) \mathrm{k} C_{56}+2 \mathrm{p}_{1} \mathrm{R}_{1} \mu_{1} \sin \left(p_{2} H\right) C_{66} \mathrm{a} \text {, } \\
& \xi_{5}=-4 \mu_{1} \mathrm{p}_{1} \mathrm{R}_{1} \cos \left(p_{2} H\right) C_{66} \mathrm{P}_{2}+\mathrm{R}_{1} \mathrm{C}_{66}^{2} \mathrm{a}^{2} \sin \left(p_{2} H\right)-4 \mathrm{i} C_{56} \mathrm{ksin}\left(p_{2} H\right) \mathrm{R}_{2}-2 C_{66} \operatorname{asin}\left(p_{2} H\right) \mathrm{R}_{2} \text {, } \\
& \left.\xi_{6}=-4 C_{66} \cos \left(p_{2} H\right) \mathrm{p}_{2} \mathrm{R}_{2}+4 \mathrm{iR}_{1} C_{56} \mathrm{ksin}\left(p_{2} H\right) C_{66} \mathrm{a}-4 \mathrm{R}_{1} \mathrm{C}_{56}^{2} \mathrm{k}^{2} \sin \left(p_{2} H\right)+4 \mathrm{R}_{1} \mathrm{C}_{56}^{2} \sin \left(p_{2} H\right) \mathrm{p}_{2}^{2}\right) \text {, } \\
& E(k)=\left(\left(4 \mathrm{i} \mu_{1} \tan \left(p_{2} H\right) \mathrm{k} C_{56}-4 \mu_{1} \tan \left(p_{2} H\right) \mu_{3} \mathrm{p}_{3}-4 \mu_{1} C_{66} \mathrm{p}_{2}+2 \mu_{1} \tan \left(p_{2} H\right) C_{66} \mathrm{a}\right) \mathrm{p}_{1}-\right. \\
& -4 \mathrm{C}_{56}^{2} \mathrm{k}^{2} \tan \left(p_{2} H\right)+4 \mathrm{iC}_{56} \mathrm{ktan}\left(p_{2} H\right) \mathrm{C}_{66} \mathrm{a}-2 \mathrm{C}_{66} \operatorname{atan}\left(\mathrm{p}_{2} \mathrm{H}\right) \mu_{3} \mathrm{p}_{3}- \\
& \left.-4 \mathrm{iC}_{56} \operatorname{ktan}\left(\mathrm{p}_{2} \mathrm{H}\right) \mu_{3} \mathrm{p}_{3}-4 \mathrm{C}_{66} \mathrm{p}_{2} \mu_{3} \mathrm{p}_{3}+4 \mathrm{C}_{66}^{2} \tan \left(\mathrm{p}_{2} \mathrm{H}\right) \mathrm{p}_{2}^{2}+\mathrm{C}_{66}^{2} \mathrm{a}^{2} \tan \left(\mathrm{p}_{2} \mathrm{H}\right)\right) \text {. }
\end{aligned}
$$




$$
\begin{aligned}
& \varphi(k-\lambda)=\left(A_{2}+A_{3}\right) \\
& A_{2}=-p_{3} \mu_{3} p_{2} B_{0}+\frac{p_{3} \mu_{3} a A_{0}}{2}+4 \mu_{3} e^{-p 3 d} p_{3}+A_{0} p_{2}^{2} C_{66}+B_{0} a C_{66} p_{2}, \\
& A_{3}=i p_{2} k C_{56} B_{0}-\frac{A_{0} a^{2} C_{66}}{4}-\frac{i k C_{56} a A_{0}}{2}+\frac{i \lambda C_{56} a A_{0}}{2}+\lambda \mu_{3} k C_{0}-\lambda k C_{55} A_{0}+\frac{2 \lambda \mu_{3} k}{p_{3} e^{p_{3} d}}-i \lambda p_{2} C_{56} B_{0} .
\end{aligned}
$$

$\underline{\text { Appendix B }}$

$$
\begin{aligned}
& P_{1}=\left(1-\frac{c^{2}}{\beta_{1}^{2}}\right)^{1 / 2} P_{2}=\left(\frac{c^{2}}{\beta_{2}^{2}}-\frac{C_{55}}{C_{66}}+\frac{C_{56}^{2}}{C_{66}^{2}}\right)^{1 / 2}, P_{3}=\left(1-\frac{c^{2}}{\beta_{3}^{2}}\right)^{1 / 2} . \\
& \xi_{7}=C_{66}\left(P_{1} \mu_{1} H_{1} k P_{2}^{2} C_{66}+P_{2}^{2} C_{66}{ }^{2}+P_{3} \mu_{3} H_{1} k P_{2}^{2} C_{66}-P_{1} \mu_{1} P_{3} \mu_{3}+P_{1} \mu_{1} H_{1} k P_{3}^{2} \mu_{3}-P_{2}{ }^{2} C_{66}{ }^{2} H_{1} k P_{3}\right), \\
& \xi_{8}=-P_{1} \mu_{1} P_{3} \mu_{3} k H_{1} C_{56}+H_{1} k C_{66}{ }^{2} P_{2}^{2} C_{56}, \\
& \xi_{9}=\left(k^{2} H_{1}^{2} P_{2}^{2} C_{66}{ }^{2}+k^{2} H_{1}^{2} P_{3}^{2} C_{66}{ }^{2}-2 k H_{1} P_{3} C_{66}{ }^{2}+C_{66}{ }^{2}-k^{2} H_{1}^{2} C_{56}{ }^{2}\right) \\
& \left.\xi_{10}=-2 k P_{3}^{2} \mu_{3}{ }^{2}+P_{2}{ }^{2} C_{66}{ }^{2}\right)\left(P_{2}{ }^{2} C_{66}{ }^{2}+\mu_{3}{ }^{2} P_{3}^{2}+P_{1}^{2} \mu_{1}^{2}{ }^{2} C_{66}{ }^{2}\right)\left(P_{2}{ }^{2} C_{66}{ }^{2}+\mu_{1}{ }^{2} P_{1}^{2}\right)\left(-1+k H_{1} P_{3}\right), \\
& \xi_{11}=C_{66} P_{2}\left(C_{66} k H_{1} P_{3}^{2} \mu_{3}+C_{66}{ }^{2} k H_{1} P_{2}{ }^{2}-P_{3} \mu_{3} C_{66}-P_{1} P_{3} \mu_{1} \mu_{3} k H_{1}-P_{1} \mu_{1} C_{66}+C_{66} k H_{1} P_{1} P_{3} \mu_{1}\right) \\
& \xi_{12}=-k H_{1} C_{56} C_{66} P_{2}\left(\mu_{1} P_{1}+\mu_{3} P_{3}\right) \\
& \xi_{13}=\left(\left(\xi_{9}+\sqrt{\xi_{9}{ }^{2}+\xi_{10}{ }^{2}}\right) / 2\right)^{1 / 2}, \xi_{14}=\xi_{10} /\left(2\left(\xi_{9}+\sqrt{\xi_{9}{ }^{2}+\xi_{10}{ }^{2}}\right)\right)^{\frac{1}{2}}, \\
& S_{1}^{\prime}=\left[\begin{array}{l}
\left.k H^{\prime} P_{1} P_{2}^{2} \mu_{1} \mu_{2}+P_{2}^{2} \mu_{2}^{2}+k H^{\prime} P_{2}^{2} \mu_{2} \mu_{3} P_{3}-P_{1} \mu_{1} \mu_{3} P_{3}+P_{1} \mu_{1} k H^{\prime} P_{3}^{2} \mu_{3}-k H^{\prime} P_{2}^{2} \mu_{2}^{2} P_{3}\right] \\
+\left\{\left(P_{2}^{2} \mu_{2}^{2}+P_{1}^{2} \mu_{1}^{2}\right)\left(k^{2} H^{\prime 2} P_{2}^{2}-2 k H^{\prime} P_{3}+k^{2} H^{\prime 2} P_{3}^{2}+1\right)\left(P_{2}^{2} \mu_{2}^{2}+P_{3}^{2} \mu_{3}^{2}\right)\right\}^{1 / 2}
\end{array}\right. \\
& T_{1}^{\prime}=\left[P_{2}\left\{k H^{\prime} P_{3}^{2} \mu_{2} \mu_{3}+k H^{\prime} P_{2}^{2} \mu_{2}^{2}-P_{3} \mu_{2} \mu_{3}-P_{1} \mu_{1} k H^{\prime} \mu_{3} P_{3}-P_{1} \mu_{1} \mu_{2}+k H^{\prime} \mu_{1} \mu_{2} P_{1} P_{3}\right\}\right]
\end{aligned}
$$

\section{References}

Achenbach J. D., 1976. Wave propagation in Elastic solids, North Holland Pub. Comp., New York.

Bhattacharya J., 1962. On the dispersion curve for Love waves due to irregularity in the thickness of the transversely isotropic crustal layer. Beitr. Geophys., Vol. 71, pp. 324-333.

Biot M. A., 1961. Mechanics of incremental deformation, John Wiley and Sons Inc., New York.

Chattopadhyay A., 1975. On the dispersion equation for Love wave due to irregularity in the thickness of the non-homogeneous crustal layer, Acta Geophys. Pol., Vol. 23, pp. 307-317.

Chattopadhyay A. and De R. K., 1983. Love wave in a porous layer with irregular interface, Int. J. Engg. Sci., Vol. 11, pp. 11951203.

Chattopadhyay A. and Pal A. K., 1983. Dispersion curves of SH waves caused by irregularity in the prestressed internal stratum, Acta Geophys. Pol., Vol. 31, No. 1, pp. 37-49.

Chattopadhyay A., Gupta S., Sharma V.K., and Pato Kumari, 2008. Propagation of SH waves in an irregular monoclinic crustal layer, Arch. Appl. Mech., Vol. 78, pp. 989-999.

Eringen A. C., and Samuels C. J., 1959. Impact and moving loads on slightly curved elastic half-space, J. Appl. Mech., Vol. 26, pp. 491-498.

Ghosh M. L., 1961. On passage of Love waves in low period range across the ocean, Beitr. Geophys., Vol. 70, pp. 319-342.

Gubbins D., 1990. Seismology and plate tectonics, Cambridge University press, Cambridge, New York, p-170.

Mal A. K., 1962. On the frequency equation for Love waves due to abrupt thickening of the crustal layer, Geofis. Pure and Appl. Vol. 52, pp. 59-68.

Sezawa K., 1935. Love waves generated from a source of a certain depth, Bull. Earthqu. Res Inst., Vol. 13, pp. 1-17. 
Sinha N., 1967. Propagation of Love waves in a non-homogeneous stratum of finite depth sandwiched between two semi-infinite isotropic media, Pure and Appl. Geophys., Vol. 67, pp. 65-70.

Tiersten H. F., 1969. Linear piezoelectric plate vibrations, Plenum Press, New York.

Tranter C. J., 1966. Integral Transform in Mathematical Physics, Methuen and Co. Ltd., pp. 63-67.

Willis H. F., 1948. A formula for expanding an integral as a series, Phil. Mag., Vol. 39, pp. 455-459.

Xue D., Betzler K. and Hesse H., 2000. Dielectric properties of lithium niobate-tantalate crystals, Solid State Communications,

Vol.115, pp. 581-585.

\section{Biographical notes}

Professor Amares Chattopadhyay is working in Indian School of Mines since 1976. He was Awarded Atomic Energy Fellowship by the Government of India during 1973-76 for Ph.D work, Post Doctoral Fellowship for research work in 'Pierre et Marie Curie University', Paris, France during 1982-1983, and INSA-Royal Society Fellowship in 1999 for research work in UK. He worked as a Visiting Professor in the TU, Vienna, Austria and also in the University of Kaiserslautern, Germany during 2002-2003. He received Michael Madhusudan Award in 2004 and Subarna and Sashibhusan Memorial Award, in 2005. Recently he became Fellow (FICDM) of a learned society.

Dr. Shishir Gupta is an Associate professor in the Department of Applied mathematics, Indian School of Mines, Dhanbad. A Gold Medalist from Ranchi University, he has had a brilliant career. He has more than 21 years of teaching experience at undergraduate and postgraduate levels in Indian School of Mines, Dhanbad. He possesses experience of guiding students of MPhil and PhD. He has published more than 45 papers in International/National journals/Proceedings. He has served as reviewer in renowned International/ National books and journals. He has also carried out several sponsored research projects.

V. K. Sharma received his M.Sc. degree from V.B.University, Hazaribag, India and M.Phil. degree from Indian School of Mines, Dhanbad, India in 2004 and 2007 respectively both in Mathematics. He is pursuing his Ph.D. in Seismology from Indian School of Mines, Dhanbad, India. Currently he is working as a Scientist in ISSA Lab, DRDO, Delhi, Ministry of Defence, India. His areas of interest include blast modeling and study of Seismic wave propagation.

Pato Kumari received her M.Sc. degree from Ranchi University, Ranchi, India and M.Phil. degree from Indian School of Mines, Dhanbad, India in 2005 and 2007 respectively both in Mathematics. She has submitted her Ph.D. thesis to Indian School of Mines, Dhanbad, India in 2009. Currently she is working as Associate Lecturer in Jaypee University of Information Technology, Waknaghat, Himachal Pradesh, India. Her areas of interest are study of Seismic wave propagation and Application of wave mechanics to Seismological problem.

Received January 2010

Accepted February 2010

Final acceptance in revised form February 2010 